\title{
BDNF genetic variants and methylation: effects on cognition in major depressive disorder
}

\author{
Alex Ferrer ${ }^{1,2}$, Javier Labad ${ }^{2,3,4}$, Neus Salvat-Pujol ${ }^{1}$, Marta Barrachina, 5,6 Javier Costas ${ }^{7}$, Mikel Urretavizcaya ${ }^{1,4,8}$, \\ Aida de Arriba-Arnau', José M. Crespo ${ }^{1,4,8}$, Carles Soriano-Mas (1) ${ }^{1,4,9}$, Ángel Carracedo ${ }^{10,11,12}$, \\ José M. Menchón (iD) ${ }^{1,4,8}$ and Virginia Soria (iD) $1,4,8$
}

\begin{abstract}
Brain-derived neurotrophic factor (BDNF) gene regulation has been linked to the pathophysiology of major depressive disorder (MDD). MDD patients show cognitive deficits, and altered BDNF regulation has a relevant role in neurocognitive functions. Our goal was to explore the association between BDNF genetic and epigenetic variations with neurocognitive performance in a group of MDD patients and healthy controls considering possible modulating factors. The sample included 134 subjects, 64 MDD patients, and 70 healthy controls. Clinical data, childhood maltreatment, and neurocognitive performance were assessed in all participants. Eleven single nucleotide polymorphisms (SNPs) and two promoter regions in the BDNF gene were selected for genotype and methylation analysis. The role of interactions between BDNF genetic and epigenetic variations with MDD diagnosis, sex, and Childhood Trauma Questionnaire (CTQ) scores was also explored. We observed significant associations between neurocognitive performance and two BDNF SNPs (rs908867 and rs925946), an effect that was significantly mediated by methylation values at specific promoter I sites. We identified significant associations between neurocognitive results and methylation status as well as its interactions with MDD diagnosis, sex, and CTQ scores. Our results support the hypothesis that BDNF gene SNPs and methylation status, as well as their interactions with modulating factors, can influence cognition. Further studies are required to confirm the effect of BDNF variations and cognitive function in larger samples.
\end{abstract}

\section{Introduction}

Major depressive disorder (MDD) is a complex and highly prevalent psychiatric disorder with a high impact on quality of life and negative effects on mood, behavior, and cognition ${ }^{1}$. Cognitive dysfunction in MDD patients is a source of disability involving deficits in visual, verbal and working memory, attention, executive function, and processing speed ${ }^{1}$.

Several neurobiological mechanisms have been involved in the pathogenesis of MDD and related cognitive

\footnotetext{
Correspondence: Virginia Soria (vsoria@bellvitgehospital.cat)

${ }^{1}$ Department of Psychiatry, Bellvitge University Hospital, Bellvitge Biomedical Research Institute (IDIBELL), Neurosciences Group - Psychiatry and Mental Health, Barcelona, Spain

2Department of Mental Health, ParcTaulí Hospital Universitari, Institut d'Investigació i Innovació Parc Taulí (I3PT), Sabadell, Spain
}

Full list of author information is available at the end of the article. phenotypes, such as the neurotrophic signaling pathway. The brain-derived neurotrophic factor (BDNF) gene codes for a neurotrophin that is highly expressed in the central nervous system (CNS), mediates a variety of neuroplasticity processes, and has relevant influences on cognition and behavior ${ }^{2}$. BDNF is produced in the CNS and released to the extracellular matrix where it can interact with its receptors, tropomyosin-related kinase receptor B (TrkB) and p75 neurotrophin receptor (p75NTR), which mediate its effects ${ }^{3}$. Blood BDNF concentrations, that have been observed to correlate with BDNF expression levels in the brain ${ }^{4}$, are lower in MDD patients than in healthy controls ${ }^{5}$.

Research on the role of $B D N F$ single-nucleotide polymorphisms (SNPs) in risk for MDD has been centered on rs6265². This SNP, located in exon IX, results in a valine- 
methionine change in the pro-region of the pro-BDNF protein, which affects $B D N F$ regulation. Overall, inconclusive results have been found, with positive data showing an association between the minor allele "A" and $\mathrm{MDD}^{6,7}$ and a lack of robust association in a recent metaanalysis ${ }^{8}$. The inconsistency of these results might be explained by methodological differences, heterogeneity of MDD clinical subtypes, the influence of modulating environmental factors and/or by epigenetic mechanisms. For instance, previous studies suggest that the Met allele of rs6265 moderates the relationship between stressful life events in childhood and depression ${ }^{9,10}$. In addition, rs6265 has been also associated with brain volumes ${ }^{11}$, presence of psychosis and suicidal behavior ${ }^{12}$, tendency to chronicity $^{13}$ and treatment response in melancholic depression ${ }^{14}$.

Since $B D N F$ is involved in processes such as neuronal survival, neurogenesis and long-term potentiation ${ }^{15}$, animal studies have reported a relevant role for rs6265 in motor learning and long-term memory ${ }^{16}$, which could be related to changes in hippocampal synaptic plasticity via downregulation of 5-HT3a receptors in rs6265Met carriers ${ }^{17}$. Human clinical studies have also reported significant associations between the BDNF genotype and cognitive performance ${ }^{18,19}$, although the results are often inconsistent and meta-analyses have been negative ${ }^{20}$.

Genome-wide association studies are beginning to identify associations of $B D N F$ with several behavioral and cognitive traits $^{21}$, such as smoking initiation ${ }^{22}$, educational attainment ${ }^{23}$, highest math class $\operatorname{taken}^{23}$, and the "worry" genetic-defined subcluster of neuroticism ${ }^{24}$.

DNA methylation is an epigenetic mechanism associated with gene silencing, although some evidence suggests that it may also be associated with active gene transcription $^{25}$. Research on methylation patterns in $B D N F$ of MDD patients has focused on promoter regions I and IV. Although most research has found that MDD patients show higher methylation levels at promoter I, some studies have reported an inverse association in severely depressed patients ${ }^{26}$. Research on methylation of promoter IV has also shown increased methylation levels in patients with depression when compared to those in healthy controls ${ }^{27,28}$. Interestingly, a higher $B D N F$ promoter methylation status has been associated with suicidal ideation and suicidal attempt history in depressive patients ${ }^{29}$.

Regarding the influence of external factors on gene regulation through DNA methylation, there is evidence that DNA methylation of the $B D N F$ gene is modified by early life negative stressors ${ }^{30,31}$. Moreover, differences are observed in specific brain areas and in a sex-specific manner ${ }^{32}$. Other factors that have been observed to influence $B D N F$ methylation are tobacco consumption ${ }^{33}$, $\operatorname{age}^{34}$, and pharmacological treatment, with higher methylation levels of $B D N F$ promoter I in patients under treatment with antidepressants ${ }^{35}$.

To our knowledge, there are no previous studies addressing the involvement of BDNF regulation on cognitive performance in MDD. We hypothesize that specific methylation of promoter regions of the BDNF will be associated to poorer performance in neurocognitive tasks. Our goal was to study the association between BDNF genetic and epigenetic variation with neurocognitive performance and to explore the influence of each specific CpG site within the BDNF gene promoters on cognition, taking into account potential modulating external factors and confounders.

\section{Materials and methods Study sample}

The sample consisted of 64 MDD patients (72\% females, mean age $57.1 \pm 1.3$ years) diagnosed according to DSMIV-TR criteria (American Psychiatric Association, 2000) and 71 healthy controls (HCs) $(63 \%$ females, mean age $54.2 \pm 1.3$ years. MDD patients were recruited from the Psychiatry Department at Bellvitge University Hospital (Hospitalet de Llobregat, Barcelona) while $\mathrm{HCs}$ were recruited from the same geographic region through advertisements. All participants were unrelated Caucasians of Iberian Peninsula ancestry.

The exclusion criteria were as follows: age less than 18 years, non-Caucasian ethnicity, a diagnosis of other psychiatric disorders including substance abuse or dependence (except nicotine), neurological disorders including dementia, mental retardation, severe medical conditions, electroconvulsive therapy in the previous year, pregnancy or puerperium, and corticosteroid treatment in the previous 3 months.

The sample partially overlaps that used in previous studies $^{36,37}$, which explored different hypotheses. The current participants agreed to provide blood samples for DNA extraction, genotyping, and methylation studies. The Clinical Research Ethics Committee (CEIC) of the Bellvitge University Hospital approved the research protocol, all participants provided written informed consent after having received a full explanation of the study.

\section{Clinical assessment}

All patients met DSM-IV-TR criteria for MDD and were interviewed by experienced psychiatrists using the Mini-International Neuropsychiatric Interview (MINI) ${ }^{38}$. HCs were required to have no history of present or past psychiatric disorders and to show a score lower than 7 in the 28-item Spanish adaptation of the Goldberg General Health Questionnaire (GHQ-28). A semistructured interview was administered to all participants to asses sociodemographic and clinical variables, substance use and treatments. Antidepressant treatment was recorded 
using the World Health Organization Anatomical Therapeutic Chemical Classification System ${ }^{39}$, which measures treatment as the defined daily dose (DDD), that is, the assumed average maintenance dose per day for a drug used for its main indication in adults.

The 17-item Hamilton Depression Rating Scale (HDRS) was used to assess depression severity ${ }^{40}$. Treatment resistance in the MDD group was assessed using the staging model as proposed by Thase and Rush ${ }^{41}$, which defines 5 levels of resistance according to the number and classes of antidepressants that have failed to produce a response. The state-trait anxiety inventory (STAI) was used to evaluate anxiety both in patients and $\mathrm{HCs}^{42}$. The Childhood Trauma Questionnaire (CTQ), which examines the exposure to five types of trauma in childhood and has been previously validated in healthy and clinical populations, was administered to all participants ${ }^{43}$. Three types of childhood maltreatment are focused on abuse (sexual, physical, and emotional) while two types of maltreatment are centered on neglect (physical and emotional). Each type of maltreatment is measured using five items and each item is rated on a five-point scale.

\section{Neuropsychological assessment}

The Spanish version of the Mini-mental State Examination (MMSE) was used as a screening for dementia. The following neuropsychological tests were administered to all participants to assess different cognitive domains: (1) verbal learning and memory: Hopkins Verbal Learning Test Revised $^{\mathrm{TM}}$ (HVLT-R); (2) visual learning and memory: Brief Visuospatial Memory Test Revised ${ }^{\mathrm{TM}}$ (BVMTR) and the Rey Complex Figure Test (RCFT), which includes copy, immediate recall, and delayed recall subscores; (3) working memory: Corsi Block-Tapping Test (CBTT) and Letter-Number Span (LNS); (4) processing speed: Trail Making Test Part A (TMT-A), Brief Assessment of Cognition in Schizophrenia - Symbol Coding (BACS-SC), and category fluency (animal naming); (5) attention/vigilance: Continuous Performance Test Identical Pairs (CPT-IP);(6) selective attention/interference: Stroop test (direct subscores for words, W; colors, C; words-colors, WC; interference); (7) reasoning and problem solving: Neuropsychological Assessment Battery ${ }^{\circledast}$ Mazes (NAB-Mazes); (8) executive control: Trail Making Test Part B (TMT-B). In all tests, higher scores reflected better cognitive performance with the exceptions of TMT$A$ and TMT-B, where the outcome measure is the number of seconds needed to perform the task; thus, higher scores reflected poorer cognitive performance.

\section{SNP selection and genotyping}

Blood samples were obtained close to the neuropsychological assessment. The Tagger tool of Haploview v.4.2 $2^{44}$ was used to select tagSNPs in linkage disequilibrium (LD) $\left(r^{2}>0.7\right)$ with the remaining SNPs at minor allele frequency (MAF) $>10 \%$ from the HapMap phase III European samples. Functional SNP rs6265 and SNPs most commonly associated with mental disorders or cognitive dysfunction from the literature (rs2030324, rs12273363, rs908867, and rs1491850) were prioritized as tagSNPs ${ }^{45-48}$. In addition, rs11030094, rs11602246, and rs4923463, which were absent from the HapMap data, were included following Hennings, Honea, and Neves ${ }^{49-51}$. In total, 11 SNPs were selected (Fig. S1).

Genotyping of the selected SNPs was performed using the MassARRAYiPLEX platform (Agena Bioscience, formerly Sequenom, Inc.; San Diego, California). We used mean methylation levels from the performed technical replicas and discarded outlying values (standard deviation $>10 \%$ ) as a quality control method. The genotyping assays were performed at the genotyping facilities of CeGen in the Santiago de Compostela Node ('Centro Nacional de Genotipado').

\section{Selection of genomic regions and quantitative DNA methylation analysis}

We selected promoters I and IV for our methylation study analyses since these are promoters with more consistent associations with psychiatric disorders ${ }^{27}$. We used two assays to cover promoter I, with Assay 1 covering the region chr11: 27744025-27744278 and Assay 2 covering the region chr11: 27744414-27744653, and one assay for promoter IV, covering chr11: 2772230527722675 (UCSC h19 assembly). The CpG sites analyzed in each region can be found in Table S1 of the Supplementary Material.

DNA purification, bisulfite treatment, and quantitative DNA methylation analysis using the MassArray platform of SEQUENOM were performed as described ${ }^{52}$. Primers were designed using MethPrimer (http://www.urogene. org/ methprimer/). Primers were tagged to obtain an appropriate product for in vitro transcription, prevent abortive cycling and balance the PCR primer length (forward primer tag: cagtaatacgactcactatagggagaaggct, reverse primer tag: aggaagagag). The sequences of the primers used for amplification are shown in Table S1 of the Supplementary Material.

\section{Statistical analyses}

We processed data using SPSS 19.0 (SPSS, IBM, USA) to perform descriptive and univariate analyses comparing the MDD and HCs groups using the $\chi^{2}$ test for dichotomic categorical variables and Student's $t$-test for continuous variables. We previously analyzed the distribution of variables, and those following a skewed distribution were $\log$ transformed (ln) to approximate normality, which was the case for the HDRS and CTQ scores and two cognitive tests (TMT-A and TMT-B). 
Association analysis at the individual SNP level was performed as implemented in PLINK $1.9^{53}$ to conduct multivariate analysis based on linear regression. Statistical significance was assessed by a permutation procedure to estimate the significance of the best result $(10,000$ permutations). Three different genetic models (dominant, recessive, and additive) were considered. These analyses represent an effective number of tests of $2.2^{54}$; therefore, the results were considered experiment-wise significant if $p$ value $<0.023$ after the permutation procedure. The analyses included sex, age, years of education, MDD diagnosis, tobacco consumption, HDRS, STAI trait subscore and CTQ score as covariates.

An analysis of plausible mediation through methylation variables in the relationship between SNPs and neurocognitive variables was performed with the PROCESS macro of SPSS version 21.0, developed by Hayes ${ }^{55}$. This method uses bias-corrected bootstrap confidence intervals. The results were considered significant when the bias-corrected 95\% confidence intervals did not contain zero.

Partial correlation analyses adjusted by age, gender, and years of education were used to explore the relationship between methylation measures and cognitive performance. We conducted a stratified analysis by diagnosis (HCs vs. MDD). In the MDD group, we also included relevant clinical variables (HDRS, CTQ score, STAI trait sub-score and antidepressant treatment).

Multiple linear regression analyses were carried out in all participants to explore the association between methylation variables and neuropsychological performance, the latter being considered as dependent variables. Separate multiple regression analyses were performed for each methylation measure, each of which was considered the main independent variable. We controlled for covariates and potential confounders including sex, age, years of education, tobacco consumption, diagnostic group, HDRS, STAI-trait sub-score and CTQ score. Antidepressant treatment was not included as a covariate since this variable presents a high collinearity with MDD diagnosis. Finally, we explored the association between neuropsychological results and the interactions between methylation status with sex, CTQ score and MDD diagnosis. Standardized beta coefficients will be reported. Standardized beta coefficients are calculated by subtracting the mean from the variable and dividing by its standard deviation. This results in standardized variables having a mean of zero and a standard deviation of 1 . The standardization allows the comparison of all independent variables in the equation. Standardized beta coefficients reflect how many standard deviations a dependent variable changes, per standard deviation increase in the predictor (independent) variable. As the standardized beta coefficient reflects the strength of the association between an independent variable (e.g. methylation of the promoter
IV of the BDNF gene) and the dependent variable (e.g. visual memory task), a positive beta coefficient would indicate a better cognitive performance in those individuals with a greater methylation whereas a negative beta coefficient would indicate a poorer cognitive performance in those individuals with a greater methylation.

The main analyses testing the hypotheses related to the role of methylation of promoter I and IV of the BDNF gene (mean methylation values) on each cognitive test (17 different cognitive measures) were adjusted for multiple comparisons with the Benjamini-Hochberg procedure, that allows controlling for the false discovery rate $(\mathrm{FDR})^{56}$. The results will be adjusted for different FDR $(20 \%, 10 \%$, and $5 \%$ ) taking into account 34 comparisons (17 cognitive variables $\times 2$ promoters). We further performed statistical analyses under an exploratory approach when studying association between specific $B D N F$ methylation CpGs and cognitive performance. For these analyses, the statistical significance level was set at $p<0.05$ (bilateral), without an adjustment for multiple comparisons, as correction for multiple testing is not strictly necessary in those analyses that are exploratory in nature ${ }^{57}$.

\section{Results \\ Univariate analysis}

Demographic and clinical variables are shown in Table 1. As expected, MDD patients showed higher HDRS scores than HCs. MDD patients also showed significantly higher scores in the CTQ and STAI trait subscore. In the cognitive functioning analysis, MDD patients showed poorer cognitive performance than $\mathrm{HCs}$ on all cognitive domains (Table 2). Mean methylation values at each CpG site are shown in Table S2 of the Supplementary Material. Methylation levels were higher in HCs than in MDD with significant differences in CpG 1 and 7.8.9 of promoter I assay 1, CpG 14 of promoter I assay 2 and CpG 11, 13 and 15.16.17 of promoter IV.

\section{Association analyses of individual SNPs}

All genotyped polymorphisms were in Hardy-Weinberg equilibrium and had call rates higher than $90 \%$. After correction for multiple testing using a 10,000 permutation procedure, two SNPs showed an association with cognitive performance under a dominant model: rs908867-T (standardized $\beta$ : 0.228; $P$ value: 0.013 ) was associated with the BVMTR test outcome, and rs925946T (standardized $\beta$ : $0.165 ; P$ value: 0.008 ) was associated with the BACS-SC result. No other associations between individual SNPs and cognitive performance were detected. We also tested for possible interactions between significant SNPs with sex, CTQ and MDD diagnosis. One significant result was found between the interaction of rs925946- $\mathrm{T}$ and MDD diagnosis with the BACS-SC result (MDD diagnosis $[\beta:-0.25 ; p$ value: 0.005$]$, 
Table 1 Demographic and clinical data of the study sample

\begin{tabular}{|c|c|c|c|}
\hline & $\mathrm{HCs}(n=70)$ & $\begin{array}{l}\text { MDD } \\
(n=64)\end{array}$ & $p$ value \\
\hline Age (years) & $54.5(10.5)$ & $57.1(10.6)$ & 0.171 \\
\hline Female gender, $n(\%)$ & $44(62.9 \%)$ & $46(71.9 \%)$ & 0.267 \\
\hline Education (years) & $11.6(3.3)$ & $9.7(4.2)$ & 0.004 \\
\hline $\begin{array}{l}\text { Tobacco consumption } \\
\text { (cigarettes/day) }\end{array}$ & $2(5.3)$ & $4.1(8.6)$ & 0.089 \\
\hline HDRS & $0.7(1.1)$ & $12.3(9.1)$ & $<0.001$ \\
\hline $\begin{array}{l}\text { Antidepressant treatment } \\
\text { (DDD) }\end{array}$ & $0(0)$ & $2.3(1.3)$ & $<0.001$ \\
\hline Treatment resistance (Thase) ${ }^{a}$ & - & - & - \\
\hline Stage 0 & & $20(31.3 \%)$ & \\
\hline Stage I & & $13(20.3 \%)$ & \\
\hline Stage II & & $10(15.6 \%)$ & \\
\hline Stage III & & $12(18.8 \%)$ & \\
\hline Stage IV & & $9(14.1 \%)$ & \\
\hline Stage V & & $0(0 \%)$ & \\
\hline $\begin{array}{l}\text { Childhood trauma } \\
\text { questionnaire }^{\text {b }}\end{array}$ & $34.3(9.7)$ & $38.5(14.1)$ & 0.043 \\
\hline STAI trait score & $15.3(8.6)$ & $32.3(13.7)$ & $<0.001$ \\
\hline
\end{tabular}

All variables presented in mean (SD), or $n(\%)$. Missing data: CTQ (1.5\%), STAI (1\%)

HCs Healthy controls, MDD major depressive disorder, HDRS Hamilton Depression Rating Scale, DDD Defined Daily Doses

${ }^{a}$ Criteria for treatment resistance stages were: non-resistant or any medication trials, to date, judged to be adequate (Stage 0); failure of at least one adequate trial of one major class of antidepressant (Stage 1); failure of at least two adequate trials of at least two distinctly different classes of antidepressants (Stage II); Stage II resistance plus failure of an adequate trial of a tricyclic antidepressant or a first augmentation strategy (lithium or thyroid hormone) (Stage III); Stage III resistance plus failure of an adequate trial of an MAOI or a second augmentation strategy (Stage IV); Stage IV resistance plus failure of an adequate course of bilateral electroconvulsive therapy (Stage V)

rs925946 [ $\beta$ : 0.081; $p$ value: 0.226$]$, interaction MDD $\mathrm{x}$ rs925946-T $[\beta: 0.171 ; p$ value: 0.035$])$. We also performed post hoc analyses of the association between individual SNPs and treatment resistance in the MDD group but found no significant association.

\section{Mediation analysis}

A significant mediation was found in the association between rs908867 and the BVMTR test results through differential methylation at the CpG 7.8.9 site of promoter I. The bias-corrected bootstrap 95\% CI indicated that the indirect effect through methylation differences was significant using a dominant model (Effect: 0.84 (SE 0.49), $95 \%$ CI, $[0.15,2.24])$. No other significant mediations were found.
Table 2 Neuropsychological performance in major depressive disorder patients and healthy controls

\begin{tabular}{|c|c|c|c|}
\hline & HCs $(n=70)$ & $\operatorname{MDD}(n=64)$ & $p$ value \\
\hline \multicolumn{4}{|l|}{ Cognitive domains } \\
\hline \multicolumn{4}{|l|}{ Verbal learning and memory } \\
\hline HVLT-R & $23.86(4.92)$ & $20.37(5.17)$ & $<0.001$ \\
\hline \multicolumn{4}{|l|}{ Visual learning and memory } \\
\hline BVMT-R & $22.66(7.52)$ & $14.72(9.08)$ & $<0.001$ \\
\hline RCFT - copy & $30.84(5.34)$ & $27.70(7.42)$ & 0.006 \\
\hline RCFT - immediate recall & $17.45(6.36)$ & $12.66(6.57)$ & $<0.001$ \\
\hline RCFT - delayed recall & $17.76(6.31)$ & $12.13(6.78)$ & $<0.001$ \\
\hline \multicolumn{4}{|l|}{ Working memory } \\
\hline CBTT (non-verbal) & $14.45(3.74)$ & $11.59(3.76)$ & $<0.001$ \\
\hline LNS (verbal) & $13.72(3.41)$ & $10.58(3.49)$ & $<0.001$ \\
\hline \multicolumn{4}{|l|}{ Processing speed } \\
\hline TMT-A (seconds) & $45.71(24.00)$ & $65.76(38.41)$ & $<0.001$ \\
\hline BACS-SC & $47.73(13.8)$ & $33.11(15.89)$ & $<0.001$ \\
\hline Category fluency & $23.74(6.53)$ & $20.05(6.26)$ & 0.001 \\
\hline Stroop Direct W & $100.90(15.81)$ & $92.83(22.67)$ & 0.021 \\
\hline Stroop Direct $C$ & $70.93(12.17)$ & $61.17(14.68)$ & $<0.001$ \\
\hline \multicolumn{4}{|l|}{ Attention/vigilance } \\
\hline CPT-IP & $2.52(0.87)$ & $1.99(0.88)$ & 0.002 \\
\hline \multicolumn{4}{|l|}{ Executive function } \\
\hline TMT-Ba (seconds) & $82.24(48.83)$ & $149.19(124.23)$ & $<0.001$ \\
\hline NAB-Mazes & $14.80(6.86)$ & $8.95(7.19)$ & $<0.001$ \\
\hline Stroop Direct WC & $45.06(12.76)$ & $36.92(11.04)$ & $<0.001$ \\
\hline Stroop Direct Interference & $3.62(9.59)$ & $0.38(6.87)$ & 0.026 \\
\hline
\end{tabular}

All variables presented in mean (SD), or $n(\%)$. Missing data differed for cognitive tests: HVLT-R (0.7\%), BVMT-R (2.2\%), LNS (4.4\%), TMT A (1.5\%), BACS-SC (1.5\%), Category fluency (0.7\%), Stroop Direct W and C (0.7), CPT-IP (13.4\%), TMT-B (4.5\%), NAB-Mazes (2.2\%), Stroop Direct WC and Interference (1.5\%)

HCs healthy controls, MDD major depressive disorder, HVLT-R Hopkins Verbal Learning Test-Revised, BVMT-R Brief Visuospatial Memory Test-Revised, RCFT Rey Complex Figure Test, CBTT Corsi Block-Tapping Test, LNS Letter Number Span, TMT-A Trail Making Test part A, BACS-SC Brief Assessment of Cognition in Schizophrenia-Symbol Coding, $W$ words, $C$ colors, CPT-IP Continuous Performance Test-Identical Pairs, TMT-B Trail Making Test part B, NAB-Mazes Neuropsychological Assessment Battery-Mazes, WC words-colors

aTMT-A and TMT-B raw scores are shown. $P$ values calculated upon natural logtransformed variables

\section{Partial correlation analyses}

We analyzed the correlation between methylation variables and neuropsychological performance. The correlation heat map of these partial correlation analyses stratified by diagnosis is included in Figs. S2 and S3 of the Supplementary Material. Antidepressant treatment was not correlated with methylation measures. 


\section{Multiple linear regression analyses}

The results of multiple linear regression analyses of mean methylation levels and neuropsychological performance in all participants are shown in Table 3.

Table 3 Results of multiple linear regression analyses of mean methylation levels and neuropsychological performance in all participants

\begin{tabular}{|c|c|c|c|c|}
\hline & \multicolumn{3}{|c|}{ Promoter I } & \multirow{2}{*}{$\begin{array}{l}\text { Promoter IV } \\
\text { Mean } \\
\beta\end{array}$} \\
\hline & $\begin{array}{l}\text { Mean } \\
\beta\end{array}$ & $\begin{array}{l}\text { Assay } 1 \\
\beta\end{array}$ & $\begin{array}{l}\text { Assay } 2 \\
\beta\end{array}$ & \\
\hline \multicolumn{5}{|c|}{ Verbal learning and memory } \\
\hline HVLT-R & 0.047 & 0.100 & -0.030 & -0.013 \\
\hline \multicolumn{5}{|l|}{ Visual learning and memory } \\
\hline BVMT-R & 0.105 & -0.042 & -0.144 & $-0.152^{*}$ \\
\hline RCFT- copy & 0.039 & 0.102 & -0.047 & -0.111 \\
\hline RCFT - immediate recall & -0.060 & $<0.001$ & -0.108 & $-0.240^{* *}$ \\
\hline RCFT - delayed recall & -0.031 & -0.009 & -0.046 & $-0.261^{* * *}$ \\
\hline \multicolumn{5}{|l|}{ Working memory } \\
\hline CBTT (non-verbal) & 0.089 & 0.083 & 0.065 & -0.014 \\
\hline LNS (verbal) & $-0.193^{* *}$ & -0.129 & $-0.207^{* *}$ & $-0.177^{*}$ \\
\hline \multicolumn{5}{|l|}{ Processing speed } \\
\hline TMT - A & -0.011 & -0.045 & 0.031 & 0.106 \\
\hline BACS SC & 0.002 & -0.022 & 0.029 & -0.048 \\
\hline Fluency & -0.014 & -0.029 & 0.009 & $-0.175^{*}$ \\
\hline Stroop Direct W & 0.049 & 0.080 & -0.004 & 0.068 \\
\hline Stroop Direct C & 0.054 & 0.059 & 0.029 & -0.029 \\
\hline \multicolumn{5}{|l|}{ Attention/vigilance } \\
\hline CPT-IP & 0.032 & 0.069 & -0.019 & -0.046 \\
\hline \multicolumn{5}{|l|}{ Executive function } \\
\hline TMT- B & -0.069 & -0.053 & -0.066 & 0.061 \\
\hline NAB Mazes & 0.006 & 0.026 & -0.019 & -0.124 \\
\hline Stroop Direct WC & 0.105 & 0.087 & 0.091 & $<0.001$ \\
\hline $\begin{array}{l}\text { Stroop Direct } \\
\text { Interference }\end{array}$ & 0.107 & 0.062 & 0.125 & -0.010 \\
\hline
\end{tabular}

Statistically significant results are highlighted as bold $\left({ }^{*} p<0.05 ;{ }^{* *} p<0.01\right.$ $\left.{ }^{* * *} p<0.001\right)$.

Linear regressions were adjusted by sex, age, years of education, MDD diagnosis, tobacco consumption, HDRS, STAI trait score and CTQ score.

$\beta$ Standardized beta coefficient, HVLT-R Hopkins Verbal Learning Test-Revised, $B V M T-R$ Brief Visuospatial Memory Test-Revised, RCFT Rey Complex Figure Test CBTT Corsi Block-Tapping Test, LNS Letter Number Span, TMT-A Trail Making Test part A, BACS-SC Brief Assessment of Cognition in Schizophrenia-Symbol Coding, $W$ words, $C$ colors, CPT-IP Continuous Performance Test-Identical Pairs, TMT- $B$ Trail Making Test part B, NAB-Mazes Neuropsychological Assessment BatteryMazes, WC words-colors, MDD major depressive disorder, HDRS Hamilton Depression Rating Scale, STAl State-Trait Anxiety Inventory, CTQ Childhood Trauma Questionnaire

\section{Promoter I}

A higher promoter I mean methylation level was associated with poorer performance in the LNS test, a result which remained significant with a FDR of $20 \%$ but did not reach significance at a FDR of $10 \%$. This association was also observed when analyzing methylation levels in the region covered at Assay 2, while it did not appear in Assay 1. The results of the multiple linear regression analyses of each CpG site in promoter I can be found in Tables S3 and S4 of the Supplementary Material. In Assay 1, CpG site 7.8.9 was associated with poor results in most cognitive domains (visual learning and memory, working memory, processing speed, attention/vigilance, and executive functioning). An inverse relationship was observed in CpG site 10, where higher methylation predicted better results in visual learning and memory, processing speed, attention/vigilance, and executive functioning tests. In Assay 2, poorer cognitive functioning in the LNS test was related to higher mean methylation levels at CpG sites 3.4.5.6 and 9. Another significant association was observed between higher methylation values at the CpG 11 site and poor performance in the BVMT-R test.

\section{Promoter IV}

Mean methylation levels at this promoter location were significantly associated, at a FDR of $20 \%$, to poorer performance in BVMT-R, RCFT immediate and delayed recall, LNS and fluency tests. However, at a 5\% FDR only RCFT immediate and delayed recall were significant. Results of multiple linear regression analyses of each CpG site in promoter IV can be found in Table S5 of the Supplementary Material. CpG sites 5, 9, and 10 presented an association with poorer cognition in several cognitive domains (verbal and visual learning and memory, working memory, processing speed and executive functioning). Additionally, CpG site 13 showed an especially significant association with worse performance in visual learning and memory tasks (RCFT copy, immediate, and delayed recall).

\section{Interaction analysis}

The results of the analyses of significant interactions between methylation variables and sex, CTQ score and MDD diagnosis are shown in Table S6 of the Supplementary Material. Regarding sex, the most consistent interaction was found between CpG site 22.23 of promoter IV, in which higher methylation values were associated with better cognitive performance in women, whereas an inverse association was observed in men. Regarding interactions between methylation variables and CTQ score, most significant interactions suggested that a combination of higher methylation and a greater history of childhood trauma were associated with poorer cognitive functioning in attention, executive function and visual 
and verbal memory. Finally, when exploring the interaction with MDD diagnosis, we found that in both promoter I and IV and in different cognitive domains, significant associations between higher methylation and the presence of MDD diagnosis could be found, where higher methylation values were associated with poorer cognition in MDD patients, while the inverse association was found in $\mathrm{HCs}$.

\section{Discussion}

We have studied the contribution of $B D N F$ genetic and epigenetic variations to cognitive functioning in MDD patients and HCs. Our main findings include the association between two SNPs as well as promoter methylation levels with several cognitive tasks and statistically significant interactions with sex, CTQ scores and MDD diagnosis. In addition, differential methylation at the $\mathrm{CpG}$ 7.8.9 site of BDNF promoter I mediated the association between the individual SNP rs908867 and visual memory.

In the present study exploring the relationship between 11 BDNF SNPs and cognitive functioning, two noncoding SNPs (rs908867 and rs925946) were associated with poorer performance on cognitive tasks dealing with visual learning, memory and processing speed. Although most research has focused on the role of the functional SNP rs6265 in cognitive performance, there is evidence that SNPs located in noncoding regions can also influence gene regulation ${ }^{58}$. In this line, a well-characterized noncoding SNP within $B D N F$ is rs12273363, which has been shown to regulate $B D N F$ transcription by altering promoter IV activity ${ }^{59}$. Although this particular SNP was not associated in our sample, our findings related to associations with two noncoding SNPs (rs908867 and rs925946) lend support to the hypothesis that noncoding SNPs may also influence $B N D F$ activity. Several associations have been found relating $B D N F$ SNP rs908867 with a variety of clinical and neuroimaging phenotypes, including suicidality $^{60}$, treatment response in $\mathrm{MDD}^{48}$ and hippocampal and cerebral atrophy ${ }^{49}$. Interestingly, in the study by Januar and colleagues ${ }^{27}$, only major homozygous patients showed an association between $B D N F$ promoter methylation and depression, and for CpG site 7.8.9, a trend association was observed with depression, with rs908867 significantly modifying this relationship. Our results support the evidence of a relationship between rs908867 and clinical phenotypes, including cognitive dysfunction, an effect that seems to be mediated by methylation at the CpG 7.8.9 site of BDNF promoter I. One study including healthy individuals found an association between rs925946 and impairments in long-term visual memory tasks ${ }^{61}$. Future studies focusing on the influence of these SNPs on $B D N F$ transcription regulation are needed to describe the pathway through which they could be involved in cognitive function.
Exploring the relationship between $B D N F$ gene regulation through methylation and neurocognitive performance showed that, while higher methylation levels of most regions were associated with poorer cognitive performance in the whole sample and across all cognitive domains, the effect of methylation in certain areas, such as CPG site 10 in promoter I, was associated with better cognitive function. These results suggest that hypermethylation at CpG site 10 could have a protective neurocognitive effect. Another interesting association was found in the promoter IV region, in which CPG site 13 showed a strong and specific association with impaired visual learning and memory, while CpG sites 3, 9, and 10 seemed to be associated with deficits in multiple areas. The mechanism through which differential methylation of CpG site 13 can have a specific impact on visual learning and memory, while other sites have a broader cognitive effect, should be the focus of future studies. Taken as a whole, our findings suggest an effect of $B D N F$ gene promoter methylation and neurocognitive performance. Our results are in accordance with previous data from epigenome-wide meta-analyses that reported an association between DNA methylation in different genomic areas and cognitive performance ${ }^{62}$.

To date, many studies have focused on the biological mechanisms through which $B D N F$ expression can influence cognitive performance. Previous studies based on animal models examined the influence of $B D N F$ variations on different cognitive processes ${ }^{63-66}$. Some studies have shown an association between $B D N F$ gene methylation and an increased risk of developing neurodegenerative disorders $^{67,68}$, but the results were not replicated in others ${ }^{69}$.

The cognitive tasks most frequently associated with methylation variables in our results are related to memory processes (visual, verbal, and working memory), which are thought to be mediated by the hippocampus and prefrontal cortex. The mechanisms that regulate hippocampal function in memory have been widely studied. Among other factors, $B D N F$ is a key protein involved in hippocampal neurogenesis ${ }^{70,71}$. BDNF function is subject to epigenetic control, which is sensitive to external modulating factors, including stress and childhood trauma $^{72}$. In this regard, animal studies have shown that adolescent trauma is associated with elevated corticosterone levels and lower levels of $B D N F$ in the dorsal hippocampus ${ }^{73}$. DNA methylation represents an epigenetic mechanism that contributes to the regulation of $B D N F$ transcription in the CNS and is therefore involved in memory formation ${ }^{66}$. Research focusing on the influence of $B D N F$ methylation and structural brain variations in MDD patients has described associations with cortical thickness, particularly in the prefrontal and occipital $\operatorname{areas}^{74}$, hippocampal atrophy ${ }^{49}$ and white matter integrity in the anterior corona radiata ${ }^{75}$. 
Our results also show that the association between $B D N F$ methylation and cognition can be moderated by sex, childhood trauma or MDD diagnosis. In reference to sex differences, previous human studies ${ }^{76}$ have reported higher levels of DNA methylation of promoter IV of $B D N F$ in females. In line with these findings, animal studies exploring whether maltreatment or nurturing care is associated with differential methylation of BDNF within the medial prefrontal cortex of adult rats have demonstrated that methylation of DNA associated with exon IV is increased in female maltreated rats ${ }^{31}$. Interestingly, in our study, we found significant interactions between methylation values and female sex with several cognitive tasks dealing with executive function and processing speed, which are known to involve the prefrontal cortex.

No previous studies have explored the moderation effect of childhood trauma on the relationship between $B D N F$ methylation and cognitive functioning. In a recent twin study, DNA methylation in several stress-related genes, including $B D N F$, mediated the association between childhood trauma and depressive symptoms at adulthood ${ }^{77}$. Animal studies have also demonstrated that early life adversity produces persistent changes in DNA methylation of $B D N F$ that cause altered $B D N F$ gene expression in the adult prefrontal cortex ${ }^{30}$. Studies in humans have shown an effect of childhood abuse on the epigenetic regulation of hippocampal glucocorticoid receptor expression, which could lead to cognitive dysfunction ${ }^{78}$.

Although the aim of our study was not to analyze differences in $B D N F$ methylation between diagnostic groups, we observed significantly higher methylation levels at specific promoter regions of the BDNF gene in $\mathrm{HCs}$ compared to MDD patients, results which contrast with previous findings showing greater methylation in patients $^{26,27}$. MDD diagnosis moderated the association between methylation of $B D N F$ and cognitive functioning in memory and executive function, as MDD patients with increased methylation showed a poorer cognitive profile. As there are no previous studies exploring this issue in relation to cognition, it is difficult to draw definitive conclusions. Our findings suggest that methylation of $B D N F$ may contribute to the persistent cognitive deficits observed in MDD patients.

To our knowledge, the present study is the first to explore the effect of $B D N F$ regulation through DNA methylation on cognitive performance while adjusting for modulating factors in MDD patients and HCs. Our results suggest a relationship between methylation levels at specific promoter locations and cognitive function. Therefore, this work suggests that future research on $B D N F$ regulation might help identify specific groups of MDD patients who might benefit the most from intensive antidepressant treatment targeting cognitive symptoms or neurocognitive rehabilitation.
There are some limitations of our study that merit discussion. First, we need to underscore that the association analysis between specific CpG sites and cognitive performance was exploratory in nature. Thus, hypotheses generated by these results need to be tested in further confirmatory studies. Second, the small sample size of our study might have reduced the statistical power to detect small effect sizes. Third, clinical variables such as childhood trauma and anxiety questionnaires were retrospectively self-reported and could be influenced by recall bias and depressive state. Fourth, patients were recruited in a naturalistic setting, and therefore relevant differences in clinical variables such as educational level existed between groups. For this reason, we adjusted all analyses by the factors that showed significant differences between groups and that could influence gene methylation according to previous literature. For example, a recent meta-analysis reported an association between methylation at three specific genomic sites and depressive symptomatology; therefore, all analyses were adjusted by HDRS results ${ }^{79}$. Moreover, MDD patients were receiving antidepressant treatment, which has been suggested to influence DNA methylation at the BDNF gene ${ }^{35}$. However, no significant association between antidepressant treatment dose (DDD) and DNA methylation at the BDNF gene was identified in our sample. Fifth, patients recruited from a tertiary source may differ from community-based cases, which could limit the generalization of results. Finally, we assessed the levels of methylation at the $B D N F$ gene in peripheral blood cells. Although it might be argued that methylation studied in peripheral blood cells could be different than methylation in the brain, recent studies suggest that peripheral BDNF methylation closely reflects that of brain tissues ${ }^{80}$.

In summary, our study provides further insight into the relationship between $B D N F$ and cognitive performance, suggesting that methylation of the $B D N F$ gene influences hippocampus- and prefrontal cortex-mediated cognitive tasks and that female sex, childhood trauma and MDD are moderators of these associations.

\section{Acknowledgements}

This study was supported in part by grants from the Carlos III Health Institute through the Ministry of Economy and Competitiveness (PI10/01753, PIE14/ 00034 and PI15/00662), co-funded by the European Regional Development Fund (ERDF) "A way to build Europe", CIBERSAM, and the Catalan Agency for the Management of University and Research Grants (AGAUR 2017 SGR 1247) We also thank CERCA Programme/ Generalitat de Catalunya for institutional support. The genotyping and methylation services were carried out at CEGENPRB3-ISCIII and were supported by grants PT13/0001 and PT17/0019, ISCIIISGEFI/FEDER. Dr. Labad received an Intensification of the Research Activity Grant (SLT006/17/00012). Dr. Costas was supported by a Miguel Servet II contract from the Carlos III Health Institute (CPII16/00019). Dr. Soriano-Mas was supported by a Miguel Servet contract from the Carlos III Health Institute (CPII16/00048). The authors are very grateful to all of the study participants, as well as to the staff of the Department of Psychiatry at Bellvitge University Hospital who helped recruit the sample for this study. We would also like to thank Sergi Sayols from the Hospital del Mar Medical Research Institute (IMIM) 
for helping in the initial statistical analysis and the technicians from the Center for Genomic Regulation (CRG) and Centro Nacional de Genotipado (CeGen).

\begin{abstract}
Author details
${ }^{1}$ Department of Psychiatry, Bellvitge University Hospital, Bellvitge Biomedical Research Institute (IDIBELL), Neurosciences Group - Psychiatry and Mental Health, Barcelona, Spain. ${ }^{2}$ Department of Mental Health, ParcTaulí Hospital Universitari, Institut d'Investigació i Innovació Parc Taulí (I3PT), Sabadell, Spain. ${ }^{3}$ Department of Psychiatry and Legal Medicine, Universitat Autònoma de Barcelona, Barcelona, Spain. ${ }^{4}$ Centro de Investigación Biomédica en Red de Salud Mental (CIBERSAM), Carlos III Health Institute, Madrid, Spain. ${ }^{5}$ Neuropathology Group, Bellvitge University Hospital, Bellvitge Biomedical Research Institute (IDIBELL), L'Hospitalet de Llobregat, Barcelona, Spain. ${ }^{6}$ Centro de Investigación Biomédica en Red de Enfermedades Neurodegenerativas (CIBERNED), Carlos III Health Institute, Madrid, Spain. ${ }^{7}$ Grupo de Xenética Psiquiátrica, Instituto de Investigación Sanitaria de Santiago, Complexo Hospitalario Universitario de Santiago de Compostela, Servizo Galego de Saúde, Santiago de Compostela, Spain. ${ }^{8}$ Department of Clinical Sciences, School of Medicine, Universitat de Barcelona, Barcelona, Spain. ${ }^{9}$ Department of Psychobiology and Methodology of Health Sciences, Universitat Autònoma de Barcelona, Barcelona, Spain. ${ }^{10}$ Fundación Pública Galega de Medicina Xenómica, Servicio Galego de Saúde, Instituto de Investigación Sanitaria de Santiago de Compostela, Santiago de Compostela, Spain. ${ }^{11}$ Grupo de Medicina Xenómica, Universidade de Santiago de Compostela, Centro Nacional de Genotipado - Instituto Carlos III, Santiago de Compostela, Spain. ${ }^{12}$ Centro de Investigación Biomédica en Red de Enfermedades Raras (CIBERER), Carlos III Health Institute, Madrid, Spain
\end{abstract}

\section{Conflict of interest}

The authors declare that they have no conflict of interest.

\section{Publisher's note}

Springer Nature remains neutral with regard to jurisdictional claims in published maps and institutional affiliations.

Supplementary Information accompanies this paper at (https://doi.org/ 10.1038/s41398-019-0601-8).

Received: 17 February 2019 Revised: 29 June 2019 Accepted: 30 July 2019 Published online: 21 October 2019

\section{References}

1. Rock, P. L., Roiser, J. P., Riedel, W. J. \& Blackwell, A. D. Cognitive impairment in depression: a systematic review and meta-analysis. Psychol. Med. 44, 2029-2040 (2014)

2. Hing, B., Sathyaputri, L., Potash, J. B. A comprehensive review of genetic and epigenetic mechanisms that regulate BDNF expression and function with relevance to major depressive disorder. Am. J. Med. Genet. B Neuropsychiatr. Genet. 177, 143-167 (2018).

3. Thomas, K., Davies, A. Neurotrophins: a ticket to ride for BDNF. Curr. Biol. 15 R262-R264 (2005).

4. Klein, A. B. et al. Blood BDNF concentrations reflect brain-tissue BDNF levels across species. Int J Neuropsychopharmacol. 14, 347-353 (2011).

5. Molendijk, M. L. et al. Serum BDNF concentrations as peripheral manifestations of depression: evidence from a systematic review and meta-analyses on 179 associations ( $\mathrm{N}=9484)$. Mol. Psychiatry 19, 791-800 (2014).

6. Hong, C. J. et al. Association study of a brain-derived neurotrophic-factor genetic polymorphism and mood disorders, age of onset and suicidal behavior. Neuropsychobiology 48, 186-189 (2003).

7. Licinio, J., Dong, C. \& Wong, M.-L. Novel sequence variations in the brainderived neurotrophic factor gene and association with major depression and antidepressant treatment response. Arch. Gen. Psychiatry 66, 488 (2009).

8. Li, M., Chang, H. \& Xiao, X. BDNF Val66Met polymorphism and bipolar disorder in European populations: a risk association in case-control, family-based and GWAS studies. Neurosci. Biobehav. Rev. 68, 218-233 (2016).

9. Hosang, G. M., Shiles, C., Tansey, K. E., McGuffin, P. \& Uher, R. Interaction between stress and the BDNF Val66Met polymorphism in depression: a systematic review and meta-analysis. BMC Med. 12, 1-11 (2014).
10. Zhao, M. et al. BDNF Val66Met polymorphism, life stress and depression: A meta-analysis of gene-environment interaction. J Affect Disord. 227, 226-235 (2018).

11. Cardoner, N. et al. Val66Met BDNF genotypes in melancholic depression: Effects on brain structure and treatment outcome. Depress. Anxiety 30, 225-233 (2013)

12. Iga, J.-I. et al. The Val66Met polymorphism of the brain-derived neurotrophic factor gene is associated with psychotic feature and suicidal behavior in Japanese major depressive patients. Am. J. Med. Genet. B Neuropsychiatr. Genet 144B, 1003-1006 (2007).

13. Lee, $Y$. et al. Association between the BDNF Val66Met polymorphism and chronicity of depression. Psychiatry Investig. 10, 56 (2013).

14. Domschke, K. et al. Brain-derived neurotrophic factor (BDNF) gene: No major impact on antidepressant treatment response. Int J. Neuropsychopharmacol. 13, 93-101 (2010)

15. Egan, M. F. et al. The BDNF val66met polymorphism affects activity-dependent secretion of BDNF and human memory and hippocampal function. Cell $\mathbf{1 1 2}$ 257-269 (2003)

16. Lu, B., Nagappan, G., Lu, Y. BDNF and synaptic plasticity, cognitive function, and dysfunction. Handb Exp Pharmacol. 220, 223-250 (2014).

17. Hao, R. et al. BDNF val66met Polymorphism Impairs Hippocampal Long-Term Depression by Down-Regulation of 5-HT3 Receptors. Front Cell Neurosci. 11, 306 (2017).

18. Miyajima, F. et al. Brain-derived neurotrophic factor polymorphism Val66Met influences cognitive abilities in the elderly. Genes Brain Behav. 7, 411-417 (2008).

19. Harris, S. E. et al. The brain-derived neurotrophic factor Val66Met polymorphism is associated with age-related change in reasoning skills. Mol. Psychiatry 11, 505-513 (2006).

20. Mandelman, S. D. \& Grigorenko, E. L. BDNF Val66Met and cognition: all, none, or some? A meta-analysis of the genetic association. Genes Brain Behav. 11, 127-136 (2012).

21. MacArthur, J. et al. The new NHGRI-EBI Catalog of published genome- wide association studies (GWAS Catalog). Nucleic Acids Res. 45(D1), D896-D901 (2017).

22. Furberg, $\mathrm{H}$. et al. Genome-wide meta-analyses identify multiple loci associated with smoking behavior. Nat. Genet. 42, 441-447 (2010).

23. Lee, J. J. et al. Gene discovery and polygenic prediction from a genome-wide association study of educational attainment in 1.1 million individuals. Nat. Genet. 50, 1112-1121 (2018).

24. Nagel, M. et al. Meta-analysis of genome-wide association studies for neuroticism in 449,484 individuals identifies novel genetic loci and pathways. Nat. Genet. 50, 920-927 (2018)

25. Uchida, S. et al. Epigenetic status of Gdnf in the ventral striatum determines susceptibility and adaptation to daily stressful events. Neuron 69, 359-372 (2011).

26. Fuchikami, M. et al. DNA methylation profiles of the brain-derived neurotrophic factor (BDNF) gene as a potent diagnostic biomarker in major depression. PLoS One 6, 4-10 (2011).

27. Januar, V., Ancelin, M. L., Ritchie, K., Saffery, R. \& Ryan, J. BDNF promoter methylation and genetic variation in late-life depression. Transl. Psychiatry 5, e619-7 (2015).

28. Kang, $\mathrm{H}$. J. et al. Longitudinal associations between BDNF promoter methylation and late-life depression. Neurobiol. Aging 36, 1764.e1-1764.e7 (2015).

29. Kang, $\mathrm{H}$. J. et al. BDNF promoter methylation and suicidal behavior in depressive patients. J. Affect Disord. 151, 679-685 (2013).

30. Roth, T. L., Lubin, F. D., Funk, A. J. \& Sweatt, J. D. Lasting epigenetic influence of early-life adversity on the BDNF gene. Biol. Psychiatry 65, 760-769 (2009).

31. Blaze, J., Scheuing, L. \& Roth, T. L. Differential methylation of genes in the medial prefrontal cortex of developing and adult rats following exposure to maltreatment or nurturing care during infancy. Dev. Neurosci. 35, 306-316 (2013).

32. Blaze, J. \& Roth, T. L. Caregiver maltreatment causes altered neuronal DNA methylation in female rodents. Dev. Psychopathol. 29, 477-489 (2017).

33. Zeilinger $\mathrm{S}$. et al. Tobacco smoking leads to extensive genome-wide changes in DNA methylation. PLoS One 8, 63812 (2013).

34. Boks, M. P. et al. The relationship of DNA methylation with age, gender and genotype in twins and healthy controls. PLoS One 4, 21-23 (2009).

35. Carlberg, L. et al. Brain-derived neurotrophic factor (BDNF) - Epigenetic regulation in unipolar and bipolar affective disorder. J. Affect Disord. 168, 399-406 (2014). 
36. Salvat-Pujol, N. et al. Hypothalamic-pituitary-adrenal axis activity and cognition in major depression: the role of remission status. Psychoneuroendocrinology $\mathbf{7 6}$, 38-48 (2017).

37. Ferrer, A. et al. FKBP5 polymorphisms and hypothalamic-pituitary-adrenal axis negative feedback in major depression and obsessive-compulsive disorder. J Psychiatr Res. 104, 227-234 (2018).

38. Sheehan D. V. et al. The Mini-International Neuropsychiatric Interview (M.I.N.I.) the development and validation of a structured diagnostic psychiatric interview for DSM-IV and ICD-10. J. Clin. Psychiatry 59, 22-33 (1998).

39. Anatomical Therapeutic Chemical (ATC) Classification System. World Health Organization Collaborating Centre for Drug Statistics Methodology (2007).

40. Hamilton, M. A rating scale for depression. J. Neurol. Neurosurg. Psychiatry 23, 56-62 (1960).

41. Thase, M. E., Rush, A. J. When at first you don't succeed: Sequential strategies for antidepressant nonresponders. J. Clin. Psychiatry 13, $23-29$ (1997).

42. Spielberger, C. D., Gorsuch, R. L., Lushene, P. R., Vagg, P. R. \& Jacobs, A. G. Manual for the State-Trait Anxiety Inventory (Form Y). https://doi.org/10.1007/ 978-1-4419-9893-4 (1983).

43. Bernstein, D. P. et al. Development and validation of a brief screening version of the Childhood Trauma Questionnaire. Child Abuse Negl. 27, 169-190 (2003).

44. Barrett, J. C., Fry, B., Maller, J. \& Daly, M. J. Haploview: Analysis and visualization of LD and haplotype maps. Bioinformatics 21, 263-265 (2005).

45. Lin, Y., Cheng, S., Xie, Z., Zhang, D. Association of rs6265 and rs2030324 polymorphisms in brain-derived neurotrophic factor gene with Alzheimer's disease: a meta-analysis. PLoS ONE 9, e94961 (2014).

46. Liu, L. et al. Evidence of association between brain-derived neurotrophic factor gene and bipolar disorder. Psychiatr. Genet. 18, 267-274 (2008).

47. Real, E. et al. A brain-derived neurotrophic factor haplotype is associated with therapeutic response in obsessive-compulsive disorder. Biol. Psychiatry 66, 674-680 (2009).

48. Gratacòs, M. et al. A brain-derived neurotrophic factor (BDNF) haplotype is associated with antidepressant treatment outcome in mood disorders. Pharmacogenomics J. 8, 101-112 (2008).

49. Honea, R. A. et al. Characterizing the role of brain derived neurotrophic factor genetic variation in Alzheimer's disease neurodegeneration. PLoS One 8, 1-9 (2013).

50. Hennings, J. M. et al. Possible associations of NTRK2 polymorphisms with antidepressant treatment outcome: findings from an extended tag SNP approach. Plos One 8, 1-12 (2013).

51. Neves, F. S. et al. The role of BDNF genetic polymorphisms in bipolar disorder with psychiatric comorbidities. J. Affect Disord. 131, 307-311 (2011).

52. Villar-Menéndez, I. et al. Striatal adenosine $A_{2 A}$ receptor expression is controlled by S-adenosyl-L-methionine-mediated methylation. Purinergic Signal. 10, 523-528 (2014).

53. Purcell, S. et al. PLINK: A tool set for whole-genome association and population-based linkage analyses. Am. J. Hum. Genet. 81, 559-575 (2007).

54. González, J. R. et al. Maximizing association statistics over genetic models. Genet Epidemiol. 32, 246-254 (2008).

55. Hayes, A. F., Scharkow, M. The relative trustworthiness of inferential tests of the indirect effect in statistical mediation analysis: does method really matter? Psychol Sci. 24, 1918-1927 (2013).

56. Benjamini, Y., Hochberg, B. Controlling the false discovery rate: a practical and powerful approach to multiple testing. J. R. Stat. Soc. 57, 289-300 (1995).

57. Bender, R. \& Lange, S. Adjusting for multiple testing - when and how? J. Clin. Epidemiol. 54, 343-349 (2001).

58. Andersson, R. et al. An atlas of active enhancers across human cell types and tissues. Nature 507, 455-461 (2014).

59. Hing, B. et al. A polymorphism associated with depressive disorders differentially regulates brain derived neurotrophic factor promoter IV activity. Biol. Psychiatry 71, 618-626 (2012).
60. Ropret, S., Komel, R. \& Videti, A. Neuroscience Letters Single nucleotide polymorphisms in the BDNF gene and suicide in the Slovenian sample. Neurosci. Lett. 602, 12-16 (2015).

61. Avgan, N. et al. BDNF variants may modulate long-term visual memory performance in a healthy cohort. Int. J. Mol. Sci. 18 (2017). https://doi.org/10.3390/ ijms18030655.

62. Marioni, R. E. et al. Meta-analysis of epigenome-wide association studies of cognitive abilities. Mol Psychiatry 11, 2133-2144 (2018).

63. Molteni, R., Rossetti, A. C., Savino, E., Racagni, G., Calabrese, F. Chronic mild stress modulates activity-dependent transcription of BDNF in rat hippocampal slices. Neural Plast. 2016, 2592319 (2016).

64. Sakata, K. et al. Role of activity-dependent BDNF expression in hippocampalprefrontal cortical regulation of behavioral perseverance. Proc. Natl Acad. Sci. USA 110, 15103-15108 (2013).

65. Munoz, P. C., Aspe, M. A., Contreras, L. S. \& Palacios, A. G. Correlations of recognition memory performance with expression and methylation of BDNF in rats. Biol. Res. 43, 251-258 (2010).

66. Lubin, F. D., Roth, T. L. \& Sweatt, J. D. Epigenetic regulation of BDNF gene transcription in the consolidation of fear memory. J. Neurosci. 28, 10576-10586 (2008).

67. Chang, L. et al. Elevation of peripheral BDNF promoter methylation links to the risk of Alzheimer's disease. PLoS One 9, e110773 (2014).

68. Rao, J. S., Keleshian, V. L., Klein, S. \& Rapoport, S. I. Epigenetic modifications in frontal cortex from Alzheimer's disease and bipolar disorder patients. Transl. Psychiatry 2, e132-e137 (2012).

69. Wen, $K$. et al. The role of DNA methylation and histone modifications in neurodegenerative diseases: a systematic review. PLoS One 11, e0167201 (2016).

70. Masi G., Brovedani P. The Hippocampus, neurotrophic factors and depression possible implications for the pharmacotherapy of depression. CNS Drugs 25 , 913-931 (2011).

71. Leal, G., Afonso, P. M., Salazar, I. L., Duarte, C. B. Regulation of hippocampal synaptic plasticity by BDNF. Brain Res. 1621, 82-101 (2015). https://doi.org/ 10.1016/j.brainres.2014.10.019.

72. Bennett, M. R. \& Lagopoulos, J. Progress in Neurobiology Stress and trauma: BDNF control of dendritic-spine formation and regression. Prog. Neurobiol. 112, 80-99 (2014).

73. Uys, J. D. et al. Developmental trauma is associated with behavioral hyperarousal, altered HPA axis activity, and decreased hippocampal neurotrophin expression in the adult rat. Ann. N. Y. Acad. Sci. 1071, 542-546 (2006).

74. Na, K.-S. et al. Brain-derived neurotrophic factor promoter methylation and cortical thickness in recurrent major depressive disorder. Sci. Rep. 6, 21089 (2016).

75. Choi, S. et al. Association of brain-derived neurotrophic factor DNA methylation and reduced white matter integrity in the anterior corona radiata in major depression. J. Affect Disord. 172, 74-80 (2015).

76. Ikegame, T. et al. DNA methylation analysis of BDNF gene promoters in peripheral blood cells of schizophrenia patients. Neurosci. Res. 77, 208-214 (2013).

77. Peng, H. et al. Childhood Trauma, DNA Methylation of Stress-Related Genes, and Depression: Findings From Two Monozygotic Twin Studies. Psychosom Med. 80, 599-608 (2018).

78. McGowan, P. O. et al. Epigenetic regulation of the glucocorticoid receptor in human brain associates with childhood abuse. Nat. Neurosci. 12, 342-348 (2009).

79. Jovanova, O. S. et al. DNA methylation signatures of depressive symptoms in middle-aged and elderly persons: Meta-analysis of multiethnic epigenomewide studies. JAMA Psychiatry 75, 949-959 (2018).

80. Stenz, L. et al. BDNF promoter I methylation correlates between post-mortem human peripheral and brain tissues. Neurosci. Res. 91, 1-7 (2015). 